\title{
Hyper-connectivity of the striatum related to restricted and repetitive behaviors' severity in children with ASD
}

\author{
Authors : Irene Dupong ${ }^{1,2}$ and Adriana Di Martino ${ }^{3}$ \\ ${ }^{1}$ UNIACT Lab, Psychiatry Team, NeuroSpin Neuroimaging Platform, CEA Saclay, Gif-sur-Yvette, \\ France; \\ ${ }^{2}$ Child and Adolescent Psychiatry Department, Robert Debré Hospital, Paris, France; \\ ${ }^{3}$ Autism and Social Cognition Center, Child Mind Institute, NY, NY, USA.
}

\begin{abstract}
Restricted and repetitive behaviors (RRB) are a core feature of autism spectrum disorder (ASD). But little is known about the underlying neurobiology of the disorder, preventing from having specific therapeutic targets. Based on the literature, we explored the correlates between a clinical score of RRB, using the Repetitive Behaviour Scale - Revised, and the intrinsic connectivity of seven striatal regions in a sample of 157 children with ASD. The sample was acquired from the ABIDE consortium. We found a significant correlation between the severity of our clinical scale and several cortico-striatal networks. Specifically, stronger connections were found between striatal seeds and two cortical areas, an occipital area and a frontal area in the left hemisphere. Intrinsic functional connectivity of the striatum could serve as a potential biomarker for improved detection of RRB severity.
\end{abstract}




\section{Introduction}

Restricted and repetitive behaviors (RRB) are a core feature of autism spectrum disorders (ASD). They include : stereotypies, insistence on sameness, inflexible routines, or ritualized patterns or verbal nonverbal behavior, highly restricted, fixated interests and hyper- or hypo-reactivity to sensory input or unusual interests in sensory aspects (1).

Despite an emerging number of studies on the subject, the underlying neural basis for these features remain incompletely understood. Few studies have focused on RRB, authors favoring symptoms of social impairments, the other core feature of ASD (1). To asses RRB a frequently used scale is the Repetitive Behavior Scale-Revised (RBS-R), that captures symptom scope at different ages (2).

Theoretical understanding and neuroimaging studies have associated RRB and ASD with the striatum, a subcortical structure belonging to the basal ganglia associated to a variety of functions including mouvement, learning, cognition and emotion (3-5). More precisely, the neuroimaging studies have associated RRB to striatal volume and connectivity anomalies in patients with ASD compared to typical developing controls (6-15). Without taking into account RRB, the striatum has been associated with ASD in functional connectivity studies $(16,17)$. Thus, investigating the connectivity between the striatum and other brain regions may be key to understanding the nature of restricted and repetitive behaviors in ASD.

We used resting state functional connectivity magnetic resonance imaging (MRI) to examine the intrinsic functional connectivity of striatum in an international cohort of children and adolescents with ASD. Quantifying the synchronization of blood oxygen level dependent (BOLD) signal fluctuations between different brain regions, has detected the functional networks that echo with many known features of anatomical organization in subjects with ASD, in other disorders and in healthy subjects (18-21). More precisely, studies using functional MRI (fMRI) in ASD have reported numerous abnormalities in intrinsic functional connectivity (iFC) as compared to those in typically developing controls, ranging from hypo-connectivity $(22-24)$ to hyper-connectivity $(16,25-29)$ or both $(30,31)$. These inconsistencies are attributed to the heterogeneity of the cohorts, small sample sizes, methodological variations and heterogeneous diagnostic methods.

The present study investigated iFC of the striatum, in children and adolescents with ASD, by using resting-state fMRI. We hypothesized that children with ASD would exhibit striatal connectivity patterns that correlate with clinical scores of their RRB. A further aim was to localise the areas, and correlate them with other clinical features. 


\section{Material and Methods}

\section{Sample selection}

The current sample was selected from the ABIDE II repository (32). Consistent with the primary aim of this study, we included only MRI data from individuals with a diagnosis of ASD and available RBS-R scores that met the following criteria: 1) RBS-R completed by an informant as differences in self and informant ratings may confound results (2) ; 2) mean frame-wise displacement (mFD) $<0.6 \mathrm{~mm}$ as this was 2 standard deviations (SD) from the mean of the originally selected sample $(0.172 \mathrm{~mm} \pm 0.21$ $\mathrm{mm})(33)$; 3) successful functional to anatomical registration. After taking the above steps, we confirmed that each collection presented with at least four datasets meeting these criterias. These steps yielded a final ASD sample of 157 datasets. Data selection flow and sample characteristics are detailed in Supplementary Figure 1 and Table 1, respectively.

\section{Imaging acquisition}

Given that the ABIDE II cohort results from a multi-site retrospective data aggregation, sequence parameters for anatomical and functional MRI data varied. Though all data were collected with 3 Tesla scanners. Details regarding data acquisition for each sample have been provided in the ABIDE website [http://fcon_1000.projects.nitrc.org/indi/abide] and are summarized elsewhere (32).

\section{Preprocessing}

Data were analyzed using version 0.3.9.1 of the Configurable Pipeline for the Analysis of Connectomes (C-PAC, http://fcp-indi.github.com/C-PAC) (34), which integrates tools from AFNI (http://afni.nimh.nih.gov/afni), FSL (http://fmrib.ox.ac.uk) and Advanced Normalization Tools (ANTs; http://stnava.github.io/ANTs) using Nipype (http://nipype.readthedocs.io/en/latest/). Before skull-stripping (using FSL BET), all anatomical images were spatially normalized to MNI152 2mm stereotactic space with linear and non-linear registrations using ANTs (35). For all functional images we performed site-specific slice time correction (AFNI 3dTshift commands) based on each site acquisition parameters. Motion correction was performed using the AFNI command 3 dvolreg by a two-pass procedure. In a first step, each functional volume was co-registered to the (un-aligned) mean functional image. In a second step, a new functional mean image based on the aligned images was used as the reference image. At this second stage, motion parameters based on the Friston 24-Parameter Model (six motion parameters, their values of preceding volumes, 12 squared values of these items (36)) were regressed. Nuisance regression also included the mean WM and CSF signals obtained in subject-specific masks with tissue probability thresholds of 0.96 and linear and quadratic trends. Following nuisance regression a band-pass filtering $(0.01-0.1 \mathrm{~Hz})$ was applied using the AFNI command 3dBandpass. A functional-to-anatomical co-registration was achieved by Boundary Based Registration (BBR) using FSL FLIRT (37). Spatial normalization of functional EPIs to MNI152 space was done by applying linear and non-linear transforms from ANTs. 


\section{Seed definition and analyses}

Given that the striatum's regions have heterogeneous functions, we based our seed given the striatum's iFC. At the individual level, we measured the iFC of different striatal seeds, chosen a priori as regions-of-interest. We based our masks on the seven striatal functional divisions empirically validated by Choi and colleagues (38). We selected these functional divisions over others as each of them map to known cortical networks including the visual, somato-motor, dorsal and ventral attention, limbic, fronto-parietal and default mode network (39). We included all these striatal divisions as each of the striato-cortical network they belong have been implicated in the processes affected by ASD $(25,40-43)$. The average time series of all voxels within each ROI was individually computed, and correlations between these time series and those of all other brain voxels were assessed. Whole-brain voxels were defined by a study-specific volume masks including voxels in $\mathrm{MNI}$ space present across $99 \%$ of all individual data. The resultant connectivity maps were smoothed (applied full-width at half-maximum $4 \mathrm{~mm}$ ). This resulted in individual participant-level maps of all voxels exhibiting significant iFC with each of the seven seeds.

\section{Group-level analysis}

A general linear model was fitted at each voxel that included five nuisance covariates (site, sex, demeaned age, demeanded mFD (33), and demeaned whole brain iFC values) and RBS-R Total scores as the primary covariate of interest. This analysis generated maps of regions exhibiting significant positive and negative FC for each seed. Gaussian random field theory was used for cluster-level multiple comparisons correction $(Z \geq 2.58$; $p \leq 0.05)$. In secondary analyses, group analyses were repeated using a more stringent threshold of $Z>3.1$.

\section{Post-hoc analysis}

To localise the clusters, maps were used in FSL to extract the percentage of voxel in the Talairach structural atlas and in Yeo's functional atlas.

Correlation analyses were applied to examine whether iFC within clusters was associated IQ, subscores of RBS-R, medication status (44), comorbidities and other clinical (ADOS-2 and ADI-R) indices, when data was available data. Finally, we divided the ASD group into low and high RBS-R total scores using the mean of the sample $(n=21)$ as the threshold. We also extracted iFC for the sames striatal seeds on a TDC population of 201 subjects, with the same inclusion criteria, image acquisition, preprocessing and seed definition analysis. 
bioRxiv preprint doi: https://doi.org/10.1101/2020.02.21.957993; this version posted February 24, 2020. The copyright holder for this preprint (which was not certified by peer review) is the author/funder. All rights reserved. No reuse allowed without permission.

DRAFT VERSION

\section{Results}

Our sample comprises 157 subjects ASD (mean age of 10,77 years, $76 \%$ males), supplementary characteristics are shown in Supplementary Table 1.

\section{RBS-R striatal iFC relationships}

Analyses revealed significant associations between the RBS-R scores and the coefficient of IFC between clusters and three striatal seeds. Specifically, as shown in Figure 1, RBS- R total scores were positively associated with the iFC between the striatal somato-motor seed and a cluster in the cuneal cortex (occipital cortex) on both hemispheres. Positive relationships were also observed for the iFC of the fronto-parietal and default mode striatal seeds with partially overlapping regions of the superior frontal gyrus in the left hemisphere (see Supplementary Table 2).

a)

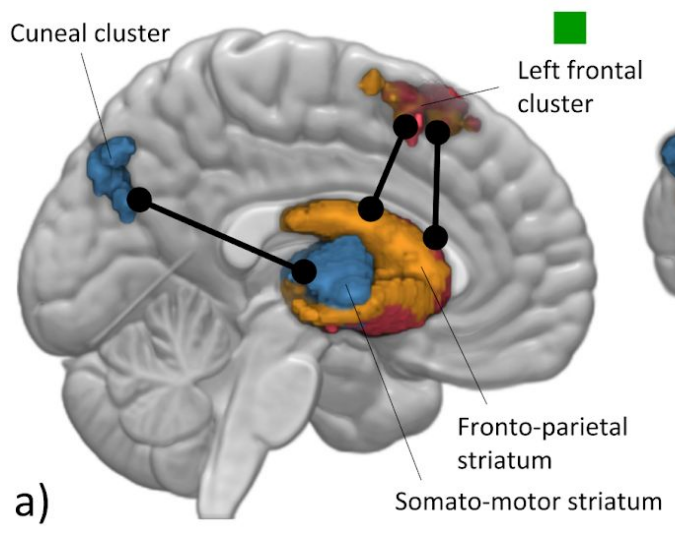

SM cluster

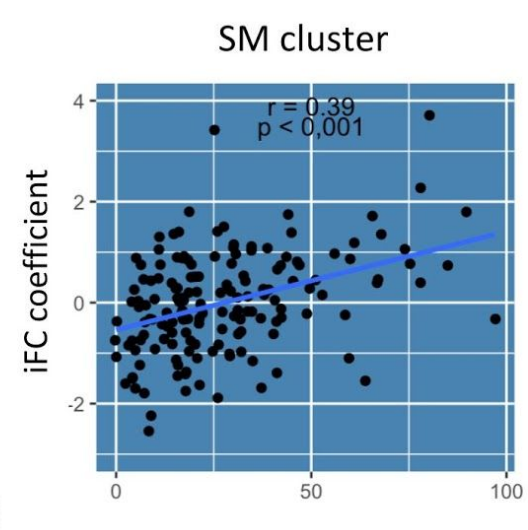

b)
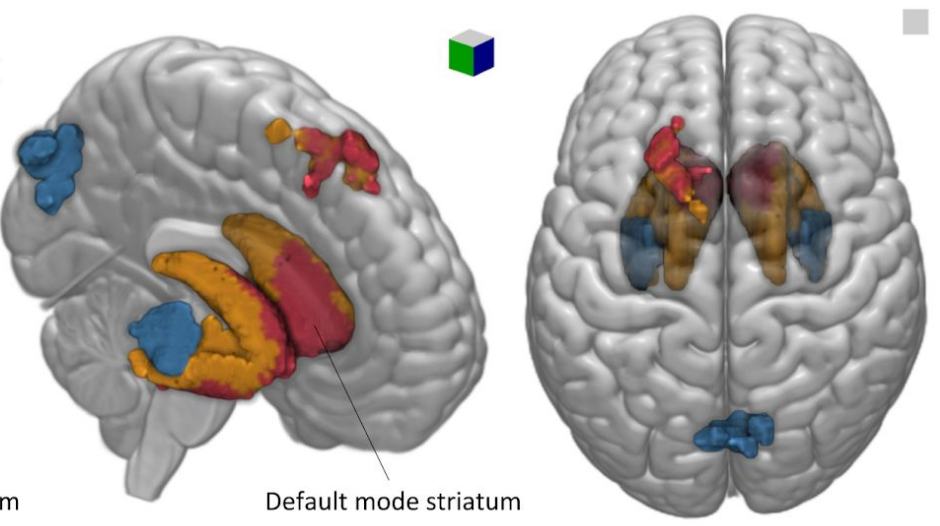

FP cluster

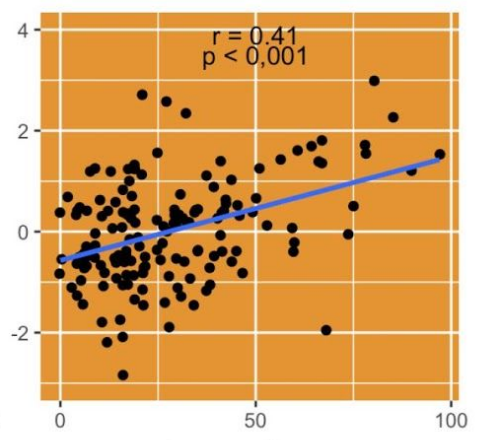

RBS-R total scores
DM cluster

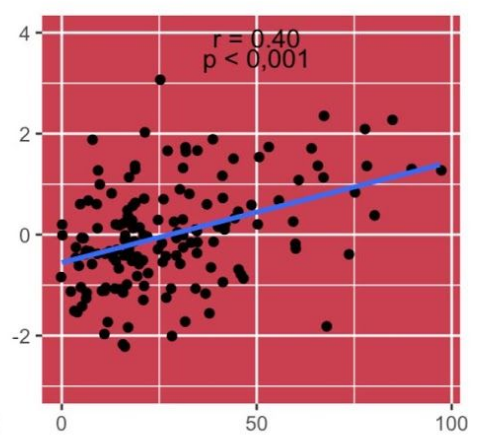

Figure 1: Correlations between significant intrinsic functional connectivity clusters ( $z$ values) and RBS-R total scores .

a) Anatomical localization of the associations

b) Scatterplots : $r$ values represent correlations between $z$ scores for each of the three clusters of significance and the RBS-R total scores for each subject.

Significant results were obtained for the same criteria with $Z=3.1$, but for a smaller cluster. 
Following Yeo's functional cortical atlas (39), the cluster associated with the SM striatal seed is distributed amongst several functional cortical areas, including mainly the visual cortex, the dorsal attention cortex, de fronto-parietal cortex and overlapping on some white matter. Whereas, the clusters associated with the FP and DM striatal seeds belong mostly to the default mode cortical area and to a lesser extent to the fronto-parietal areas. The distribution is shown in Figure 2.
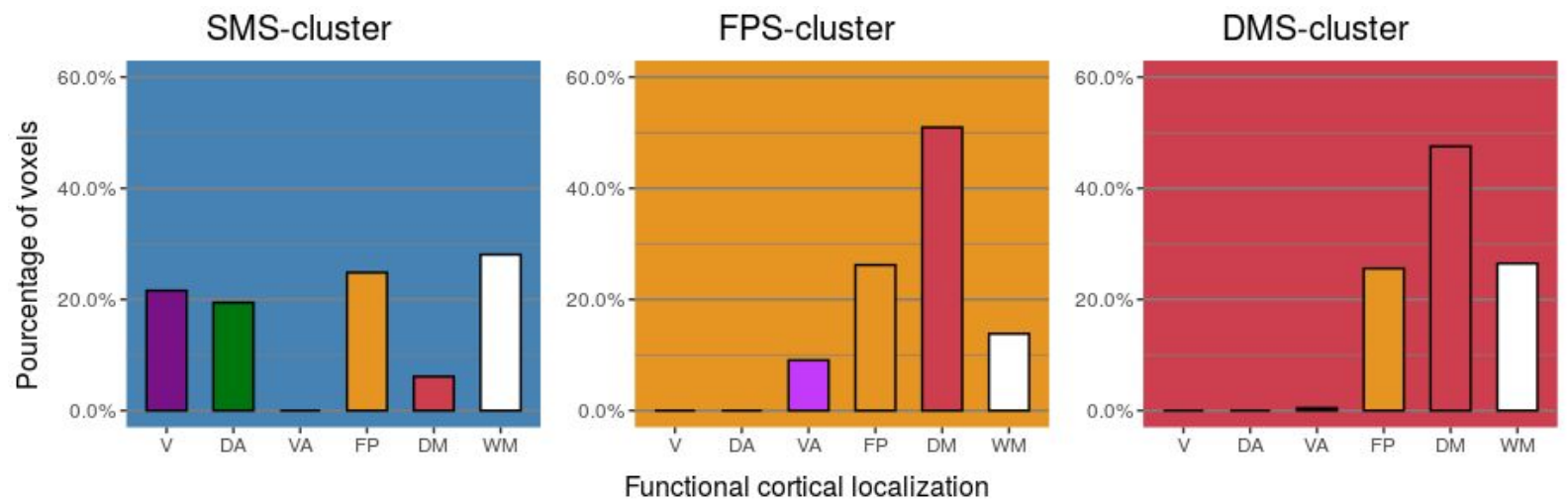

Figure 2: Distribution of each of the clusters' voxels amongst Yeo's atlas. DA : dorsal attention cortex, DM: default mode cortex, DM: default mode striatal seed, FP : fronto-parietal cortex, FPS : fronto-parietal striatal seed, SMS : somato-motor striatal seed, V : visual cortex, VA : ventral attention cortex.

\section{Correlations with clinical features}

Correlational analyses were used to examine whether connectivity between the striatal seeds and the clusters showing significant differences in regard to RBS-R total scores were influenced by intelligent quotient (IQ), comorbidity or medications.

In our sample IQ was correlated to RBS-R total scores. Global IQ was not available for all individual and was not included in the initial analysis. Is subjects with available global IQ, we used the same general linear model integrating the $z$-scores for the 3 strial seed and cluster groups, the five nuisance covariates and global IQ scores. We also integrated in another calculation the Performance $I Q$, which was available for all individuals. Then, Pearson correlations were generated between the residual iFC of each group and RBS-R total scores. As shown in Figure 3, correlation are still significant even when integrating IQ scores. 
a)

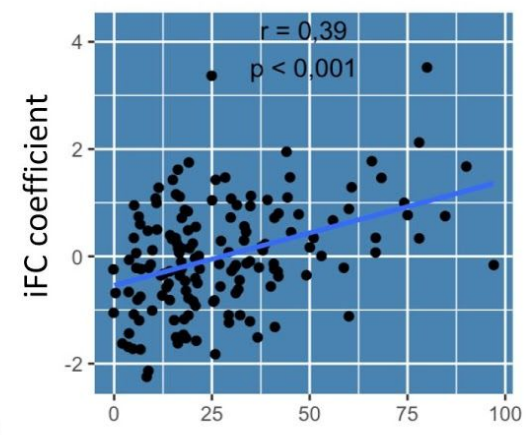

SM cluster

b)

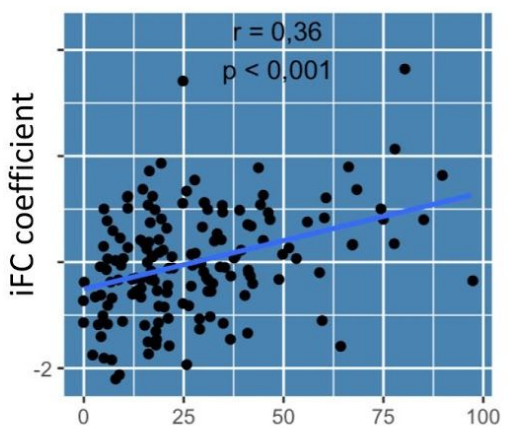

FP cluster

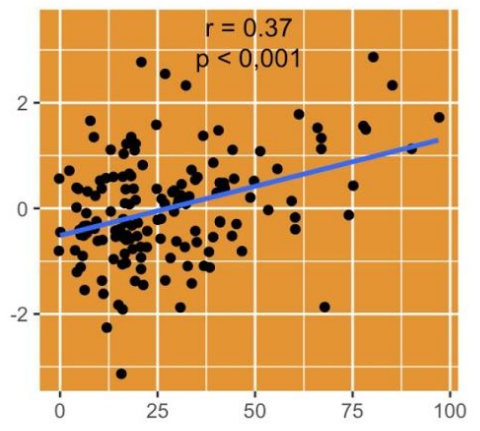

RBS-R total scores

FP cluster

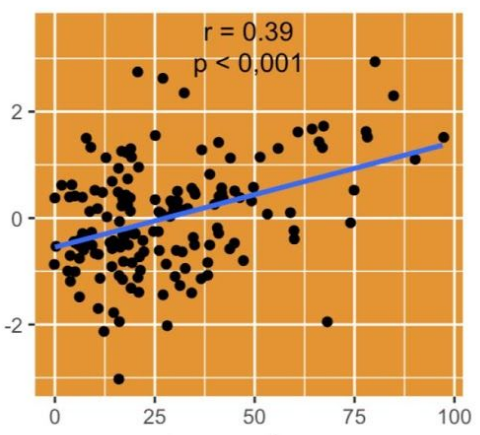

RBS-R total scores

Figure 3: Scatterplots of correlation between residual z-scores of the iFC of each seed-cluster group and RBS-R total scores, when including a) Global IQ and b) Performance IQ.

To assess if medication use and/or comorbidities had an impact on our results, we used the same model previously used but instead of including $I Q$ we included the medication and/or comorbidity status. As shown in figure 4, RBS-R total scores are still significantly correlated with the iFC of each seed-cluster when considering medication and/or comorbidity, using Pearson correlations. Only 101 subjects out of the 157 had information regarding comorbidities. 

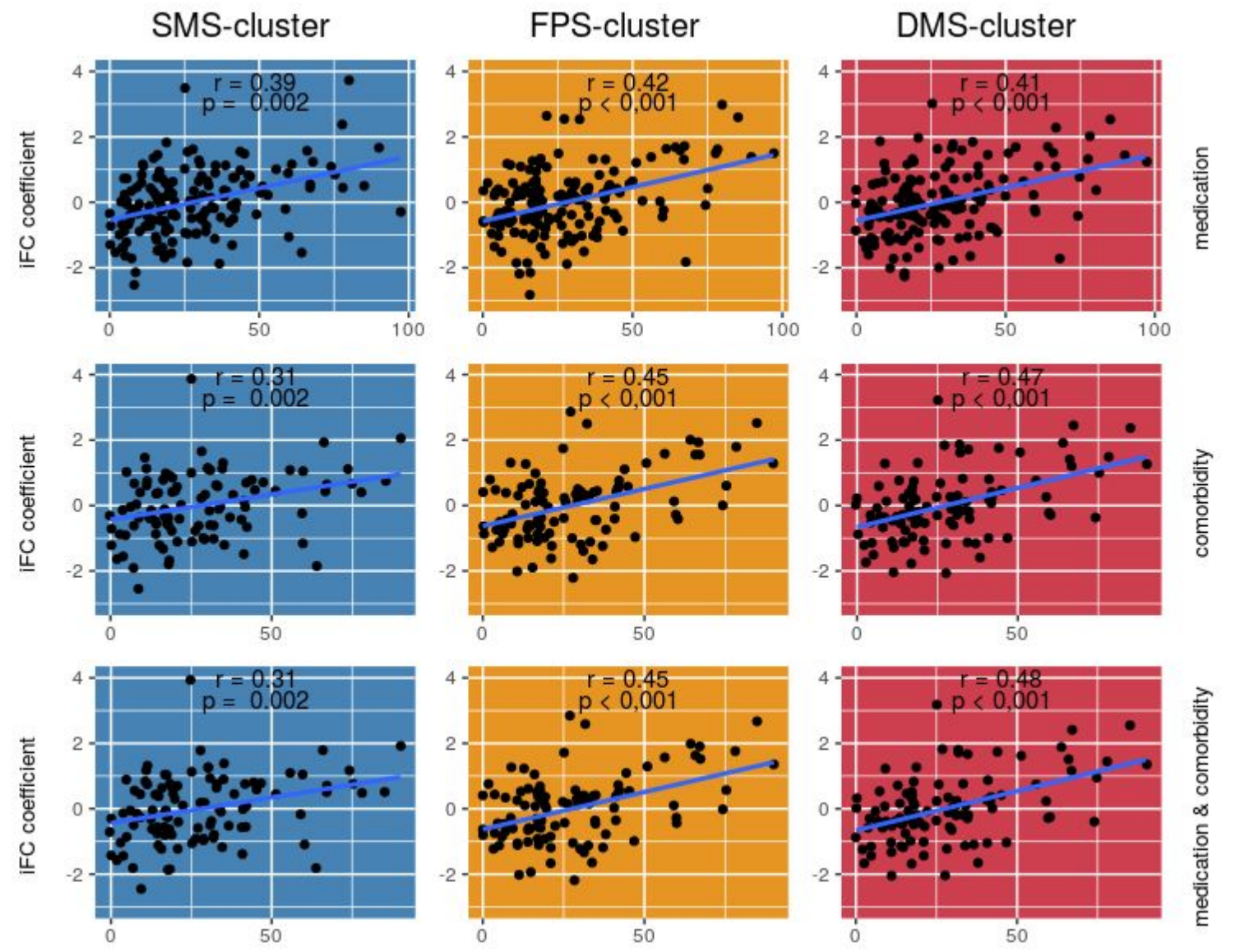

RBS-R total scores

Figure 4 : Scatterplots of correlation between residual z-scores of the iFC of each seed-cluster group and RBS-R total scores, when including a) medication use, b) comorbidities and c) medication use and comorbidities.

Correlations with other RRB scores and measures of autism symptoms (RBS-R subscores, ADOS and $A D I-R)$

First, since RBS-R total scores regroup a heterogenous set of symptoms, assessing how RBS-R subscales weighted on the correlation between RBS-R total and the iFC seemed important. Pearson correlations were generated between the residual iFC (integrating z-scores and the five nuisances) of each seed-cluster and each of the 6 RBS-R subscales (stereotyped behavior, self-injurious behavior, compulsive behavior, ritualistic behavior, insistence on sameness and restricted behavior). Five of the six subscales correlated with all the seed-cluster iFC. The subscale for self-injurious behavior was only correlated with the iFC between the DMS seed and it's cluster. The iFC between the SMS seed and it's related cluster is mainly positively correlated to sameness. Whereas the one between the FPS and DMS seeds are most correlated with compulsive behaviors. Secondly, we were interested on how the iFC was related to diagnostic assessment scales of ASD. Out of our 157 subjects, 122 subjects had total and subscores available for ADOS-2 and 127 subjects for ADI total and subscores. Again, Pearson correlations were generated between the residual iFC (integrating z-scores and the five nuisances) of each seed-cluster and domains for ADOS-2 (Social Affect subtotal, Restricted and 
Repetitive Behaviors subtotal, Severity subtotal and Total score) and ADI-R (Restricted and Repetitive Behaviors subtotal, Reciprocal Social Interaction subtotal, Verbal Communication subtotal and Non Verbal Communication subtotal). Only the activity of the DM striatal seed and it's cluster were corelated to ADI-R RRB scores. In our sample, RBS-R total scores were correlated with ADI-R RRB scores. Table 1 shows correlations between all RBS-R scores, the other ASD clinical scores and the seed-cluster iFC.

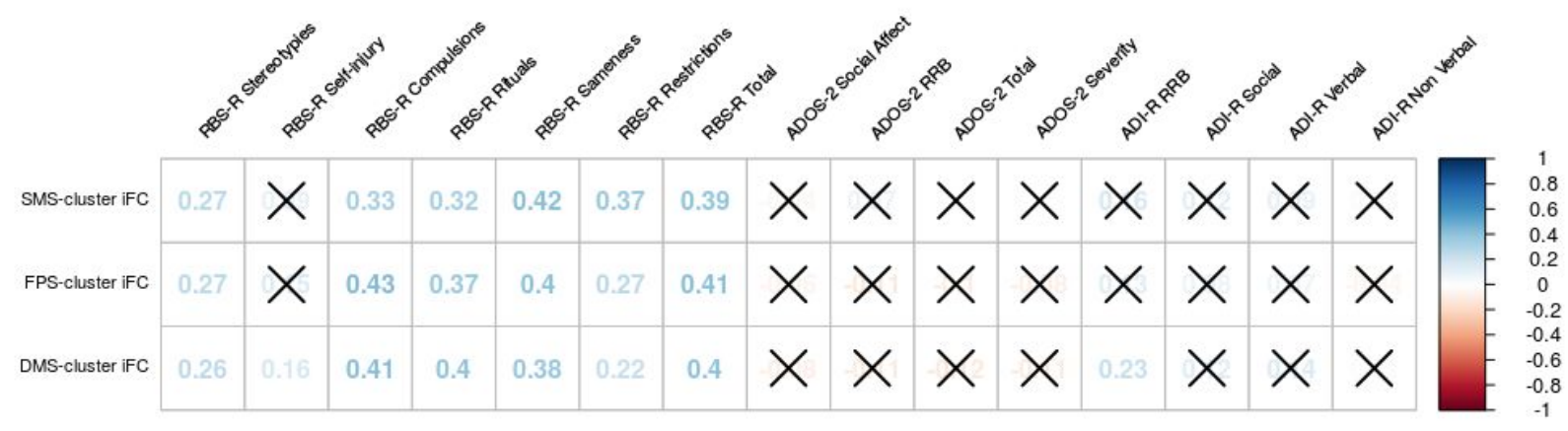

Table 1 : Correlations between residual z-scores of the iFC of each seed-cluster group and each score of the RBS-R scale as well as total and measures of ADOS-2 and ADI-R. Crossed numbers are correlations with a $p$ value $>0.05$.

High RBS-R ASD subject vs Low RBS-R ASD subject vs TDC

Finally, we divided the ASD group into low-RBS-R total scores and high-RBS-R total scores and compared there results amongst them and with the sample of TDC. Regarding the TDC sample, there were no group differences on age or handedness or eye status during the scan (opened or closed). But the samples did differ on IQ scores, as expected on RBS-R total scores and medication status. There were more males in the ASD group.

With each of these three groups, Pearson correlations were generated between the residual iFC (integrating z-scores and the five nuisances) of each seed-cluster and RBS-R total scores. As shown in Figure 5, only the High RBS-R ASD sample was significantly correlated with the all three iFC for cluster-seed groups. The low RBS-R ASD sample was only correlated with the iFC of the SM striatal seed and cluster. 

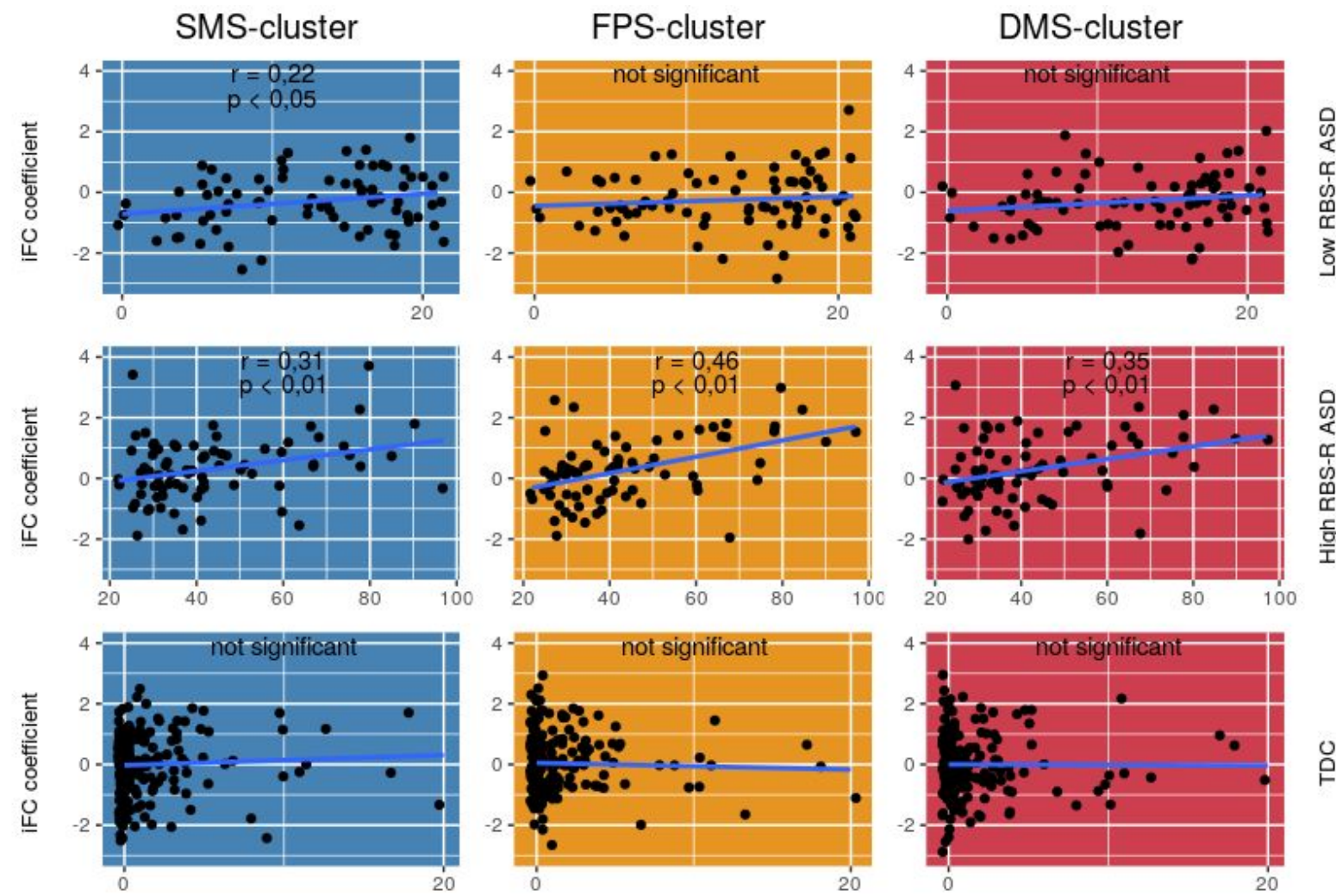

Figure 5 : Scatterplots of correlation between residual z-scores of the iFC of each seed-cluster group and RBS-R total scores for the following samples : a) Low RBS-R ASD sample, b) High RBS-R ASD sample and c) TDC sample. 


\section{Discussion}

We used resting state functional connectivity to examine the striatal functional connectivity as a function of restricted and repetitive behaviors in a 157 subject sample of 5 to 17 year-old children and adolescents with ASD. Seed based functional connectivity analyses found new evidence of a correlation between striatal connectivity and RBB. The more severe the RRB were, the stronger the connection between striatal seeds and cortical areas.

First, the more severe Total RRB scores were the stronger the connections between the striatal somato-motor seed and an occipital cortical area. Secondly, the more severe Total RRB scores were the stronger the connections between two striatal seeds, the fronto-parietal and default mode striatal seeds, and a frontal cortex area in the left hemisphere. Thirdly, these connections are not found in a sample of comparable neurotypical subjects. Chen et al. found a similar implication of regions. They linked the connectivity of these same areas as a characteristic of ASD subjects, when compared to controls using the ABIDE cohort (45). They found that out of 220 regions, the most informative connectivity was sourced in the somatosensory, default mode, visual, and subcortical regions. All regions we have more specifically linked to RRB. The association between the connectivity of striatal seeds and the cuneus cortex or the superior frontal cortex linked to RRB is a novel finding. But these regions have been independently linked to ASD or RRB.

Several limitations are to be taken into consideration. Are study excluded low functioning children with ASD, not allowing to generalize our finding to the entire population with ASD. Also, we did not apply a global signal regression, which might have maximized the information available (46). Furthermore, we used striatal seeds based on adult imaging adults when children and ASD subjects have shown a changing brain activity related to age (47). Finally, we excluded the cerebellum from our study. Cerebellum imaging needs specific setting, and several of the ABIDE site did not include the area.

Further research is needed to link RRB and their neural correlates. 


\section{Conclusion}

In sum, the current results demonstrate specific striatal functional connectivity related to the severity of restricted and repetitive behaviors in a large sample of children and adolescents with ASD. More precisely, stronger connections with occipital and frontal cortical areas were detected. These stronger connections were not found in neurotypical subjects. Intrinsic functional connectivity of the striatum could serve as a potential biomarker for improved detection of RRB severity. 


\section{References}

1. American Psychiatric Association, American Psychiatric Association, editors. Diagnostic and statistical manual of mental disorders: DSM-5. 5th ed. Washington, D.C: American Psychiatric Association; 2013. 947 p.

2. Lam KSL, Aman MG. The Repetitive Behavior Scale-Revised: Independent Validation in Individuals with Autism Spectrum Disorders. J Autism Dev Disord. 2007 May 17;37(5):855-66.

3. Alexander GE, DeLong MR, Strick PL. Parallel Organization of Functionally Segregated Circuits Linking Basal Ganglia and Cortex. Annu Rev Neurosci. 1986 Mar;9(1):357-81.

4. Schultz W, Apicella P, Scarnati E, Ljungberg T. Neuronal activity in monkey ventral striatum related to the expectation of reward. J Neurosci Off J Soc Neurosci. 1992 Dec;12(12):4595-610.

5. D. Daw N, Niv Y, Dayan P. Actions, Policies, Values, and the Basal Ganglia. Recent Breakthr Basal Ganglia Res. 2006;

6. Langen M, Bos D, Noordermeer SDS, Nederveen H, van Engeland H, Durston S. Changes in the development of striatum are involved in repetitive behavior in autism. Biol Psychiatry. 2014 Sep 1;76(5):405-11.

7. Estes A, Shaw DWW, Sparks BF, Friedman S, Giedd JN, Dawson G, et al. Basal ganglia morphometry and repetitive behavior in young children with autism spectrum disorder. Autism Res Off J Int Soc Autism Res. 2011 Jun;4(3):212-20.

8. Eisenberg IW, Wallace GL, Kenworthy L, Gotts SJ, Martin A. Insistence on sameness relates to increased covariance of gray matter structure in autism spectrum disorder. Mol Autism [Internet]. 2015 Dec [cited 2018 Mar 12];6(1). Available from: http://www.molecularautism.com/content/6/1/54

9. Sears LL, Vest C, Mohamed S, Bailey J, Ranson BJ, Piven J. An MRI study of the basal ganglia in autism. Prog Neuropsychopharmacol Biol Psychiatry. 1999 May;23(4):613-24.

10. Hollander E, Anagnostou E, Chaplin W, Esposito K, Haznedar MM, Licalzi E, et al. Striatal volume on magnetic resonance imaging and repetitive behaviors in autism. Biol Psychiatry. 2005 Aug 1;58(3):226-32.

11. Rojas DC, Peterson E, Winterrowd E, Reite ML, Rogers SJ, Tregellas JR. Regional gray matter volumetric changes in autism associated with social and repetitive behavior symptoms. BMC Psychiatry. 2006 Dec 13;6:56.

12. Wolff JJ, Hazlett HC, Lightbody AA, Reiss AL, Piven J. Repetitive and self-injurious behaviors: associations with caudate volume in autism and fragile X syndrome. J Neurodev Disord. 2013 May 2;5(1):12.

13. Uddin LQ, Supekar K, Lynch CJ, Cheng KM, Odriozola P, Barth ME, et al. Brain State Differentiation and Behavioral Inflexibility in Autism. Cereb Cortex. 2015 Dec;25(12):4740-7.

14. Abbott AE, Linke AC, Nair A, Jahedi A, Alba LA, Keown CL, et al. Repetitive behaviors in autism are linked to imbalance of corticostriatal connectivity: a functional connectivity MRI study. Soc 
Cogn Affect Neurosci. 2018 Jan 1;13(1):32-42.

15. Langen M, Leemans A, Johnston P, Ecker C, Daly E, Murphy CM, et al. Fronto-striatal circuitry and inhibitory control in autism: Findings from diffusion tensor imaging tractography. Cortex. 2012 Feb;48(2):183-93.

16. Di Martino A, Kelly C, Grzadzinski R, Zuo X-N, Mennes M, Mairena MA, et al. Aberrant Striatal Functional Connectivity in Children with Autism. Biol Psychiatry. 2011 May;69(9):847-56.

17. Delmonte S, Gallagher L, O'Hanlon E, McGrath J, Balsters JH. Functional and structural connectivity of frontostriatal circuitry in Autism Spectrum Disorder. Front Hum Neurosci [Internet]. 2013 [cited 2018 Mar 12];7. Available from: http://journal.frontiersin.org/article/10.3389/fnhum.2013.00430/abstract

18. Buckner RL, Andrews-Hanna JR, Schacter DL. The Brain's Default Network: Anatomy, Function, and Relevance to Disease. Ann N Y Acad Sci. 2008 Mar;1124(1):1-38.

19. Buckner RL, Krienen FM, Yeo BTT. Opportunities and limitations of intrinsic functional connectivity MRI. Nat Neurosci. 2013 Jul;16(7):832-7.

20. Seeley WW, Menon V, Schatzberg AF, Keller J, Glover GH, Kenna H, et al. Dissociable Intrinsic Connectivity Networks for Salience Processing and Executive Control. J Neurosci. 2007 Feb 28;27(9):2349-56.

21. Mennes M, Kelly C, Zuo X-N, Di Martino A, Biswal BB, Castellanos FX, et al. Inter-individual differences in resting-state functional connectivity predict task-induced BOLD activity. Neurolmage. 2010 May;50(4):1690-701.

22. Kana RK. Sentence comprehension in autism: thinking in pictures with decreased functional connectivity. Brain. 2006 Sep 1;129(9):2484-93.

23. Just MA. Cortical activation and synchronization during sentence comprehension in high-functioning autism: evidence of underconnectivity. Brain. 2004 Jun 16;127(8):1811-21.

24. Koshino H, Carpenter PA, Minshew NJ, Cherkassky VL, Keller TA, Just MA. Functional connectivity in an fMRI working memory task in high-functioning autism. Neurolmage. 2005 Feb;24(3):810-21.

25. Uddin LQ, Supekar K, Lynch CJ, Khouzam A, Phillips J, Feinstein C, et al. Salience network-based classification and prediction of symptom severity in children with autism. JAMA Psychiatry. 2013 Aug;70(8):869-79.

26. Lynch CJ, Uddin LQ, Supekar K, Khouzam A, Phillips J, Menon V. Default Mode Network in Childhood Autism: Posteromedial Cortex Heterogeneity and Relationship with Social Deficits. Biol Psychiatry. 2013 Aug;74(3):212-9.

27. Supekar K, Uddin LQ, Khouzam A, Phillips J, Gaillard WD, Kenworthy LE, et al. Brain Hyperconnectivity in Children with Autism and its Links to Social Deficits. Cell Rep. 2013 Nov;5(3):738-47.

28. Shih P, Keehn B, Oram JK, Leyden KM, Keown CL, Müller R-A. Functional Differentiation of Posterior Superior Temporal Sulcus in Autism: A Functional Connectivity Magnetic Resonance 
Imaging Study. Biol Psychiatry. 2011 Aug;70(3):270-7.

29. Keehn B, Shih P, Brenner LA, Townsend J, Müller R-A. Functional connectivity for an "Island of sparing" in autism spectrum disorder: An fMRI study of visual search: FCMRI for Visual Search in Autism. Hum Brain Mapp. 2013 Oct;34(10):2524-37.

30. Di Martino A, Yan C-G, Li Q, Denio E, Castellanos FX, Alaerts K, et al. The autism brain imaging data exchange: towards a large-scale evaluation of the intrinsic brain architecture in autism. Mol Psychiatry. 2014 Jun;19(6):659-67.

31. Monk CS, Peltier SJ, Wiggins JL, Weng S-J, Carrasco M, Risi S, et al. Abnormalities of intrinsic functional connectivity in autism spectrum disorders,. Neurolmage. 2009 Aug;47(2):764-72.

32. Di Martino A, O'Connor D, Chen B, Alaerts K, Anderson JS, Assaf M, et al. Enhancing studies of the connectome in autism using the autism brain imaging data exchange II. Sci Data. 2017 Mar 14;4:170010.

33. Jenkinson M, Bannister P, Brady M, Smith S. Improved Optimization for the Robust and Accurate Linear Registration and Motion Correction of Brain Images. Neurolmage. 2002 Oct;17(2):825-41.

34. Sharad S, Brian C, Ranjit K, Satra G, Chao-gan Y, Qingyang L, et al. Towards Automated Analysis of Connectomes: The Configurable Pipeline for the Analysis of Connectomes (C-PAC). Front Neuroinformatics [Internet]. 2014 [cited 2018 Feb 21];8. Available from: http://www.frontiersin.org/Community/AbstractDetails.aspx?ABS_DOI=10.3389/conf.fninf.2014.0 8.00117

35. Avants BB, Tustison NJ, Song G, Cook PA, Klein A, Gee JC. A reproducible evaluation of ANTs similarity metric performance in brain image registration. Neurolmage. 2011 Feb;54(3):2033-44.

36. Friston KJ, Williams S, Howard R, Frackowiak RS, Turner R. Movement-related effects in fMRI time-series. Magn Reson Med. 1996 Mar;35(3):346-55.

37. Greve DN, Fischl B. Accurate and robust brain image alignment using boundary-based registration. Neurolmage. 2009 Oct;48(1):63-72.

38. Choi EY, Yeo BTT, Buckner RL. The organization of the human striatum estimated by intrinsic functional connectivity. J Neurophysiol. 2012 Oct 15;108(8):2242-63.

39. Thomas Yeo BT, Krienen FM, Sepulcre J, Sabuncu MR, Lashkari D, Hollinshead M, et al. The organization of the human cerebral cortex estimated by intrinsic functional connectivity. $J$ Neurophysiol. 2011 Sep 1;106(3):1125-65.

40. Balsters $\mathrm{JH}$, Mantini $\mathrm{D}$, Wenderoth $\mathrm{N}$. Connectivity-based parcellation reveals distinct cortico-striatal connectivity fingerprints in Autism Spectrum Disorder. Neurolmage. 2018 Apr;170:412-23.

41. Farrant K, Uddin LQ. Atypical developmental of dorsal and ventral attention networks in autism. Dev Sci. 2016 Jul;19(4):550-63.

42. Kennedy DP, Courchesne E. The intrinsic functional organization of the brain is altered in autism. Neurolmage. 2008 Feb;39(4):1877-85. 
43. Padmanabhan A, Lynch CJ, Schaer M, Menon V. The Default Mode Network in Autism. Biol Psychiatry Cogn Neurosci Neuroimaging. 2017 Sep;2(6):476-86.

44. Linke AC, Olson L, Gao Y, Fishman I, Müller R-A. Psychotropic Medication Use in Autism Spectrum Disorders May Affect Functional Brain Connectivity. Biol Psychiatry Cogn Neurosci Neuroimaging. 2017 Sep;2(6):518-27.

45. Chen CP, Keown CL, Jahedi A, Nair A, Pflieger ME, Bailey BA, et al. Diagnostic classification of intrinsic functional connectivity highlights somatosensory, default mode, and visual regions in autism. Neurolmage Clin. 2015;8:238-45.

46. Murphy K, Birn RM, Handwerker DA, Jones TB, Bandettini PA. The impact of global signal regression on resting state correlations: Are anti-correlated networks introduced? Neurolmage. 2009 Feb;44(3):893-905.

47. Padmanabhan A, Lynn A, Foran W, Luna B, O’Hearn K. Age related changes in striatal resting state functional connectivity in autism. Front Hum Neurosci [Internet]. 2013 [cited 2018 Mar 12];7. Available from: http://journal.frontiersin.org/article/10.3389/fnhum.2013.00814/abstract 